\title{
GRIN2A mutations identified as key genetic drivers of epilepsy-aphasia spectrum disorders
}

T hree papers published together in Nature Genetics have highlighted mutations in GRIN2A, which encodes the NR2A subunit of the $\mathrm{N}$-methyl-D-aspartate (NMDA) receptor, as important genetic determinants of some severe forms of childhood epilepsy. "Idiopathic focal epilepsies are the most common epilepsies in childhood," says Sarah von Spiczak, who led one of the studies. "A genetic basis has been supposed, but remained unknown for the majority of patients."

Idiopathic focal epilepsies comprise a clinical spectrum ranging from benign epilepsy with controtemporal spikes (the most common childhood epilepsy, also known as rolandic epilepsy), through acquired epileptic aphasia (LandauKleffner syndrome [LKS]), to continuous spike and wave during slow-wave sleep syndrome (CSWSS) at the more severe end. LKS and CSWSS feature encephalopathy with impaired cognitive and behavioural function, including speech and language difficulties. Previous studies had suggested a link between GRIN2A mutations and some forms of epilepsy, but the precise relationship was unknown.

For their new study, von Spiczak and co-workers screened two large independent cohorts consisting of 359 patients in total, who together comprised the whole spectrum of idiopathic focal epilepsy. The cohorts were part of the EuroEPINOMICS network-a European consortium dedicated to the study of epilepsy genetics - and collaborating groups.

Mutation analysis by next-generation sequencing was performed in index patients, followed by confirmation in one cohort by Sanger sequencing, and replication in the second cohort through analysis of whole-exome data sets.

"We produced similar results in the two cohorts, and found an overall GRIN2A

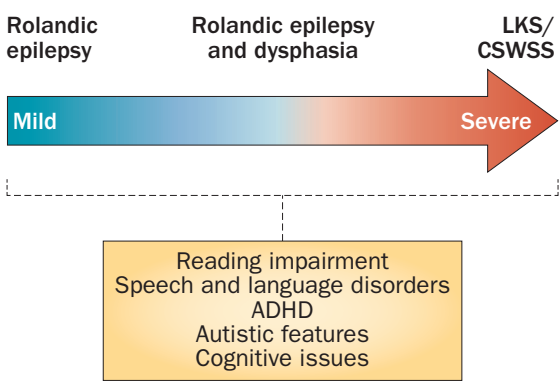

The spectrum of childhood focal epilepsies. Abbreviations: CSWSS, continuous spike and wave during slow-wave sleep syndrome; LKS, Landau-Kleffner syndrome ('acquired' epileptic aphasia). Original image courtesy of P. Szepetowski.

mutation rate of $7.5 \%$ in these patients," says von Spiczak. Mutations were more frequent in more-severe phenotypes, such as CSWSS

A second study by Pierre Szepetowski and colleagues focused on 10 families plus seven individuals with LKS or CSWSS, who were part of the French consortium known as EPILAND. The researchers screened for GRIN2A mutations in these 61 individuals by Sanger sequencing and array-based comparative genomic hybridization. "About $20 \%$ of cases were found to have a genetic origin sustained by de novo or inherited mutations in GRIN2A," says Szepetowski.

To investigate the effects of GRIN2A mutations on NMDA receptor function, the researchers transfected HEK293T cells with wild-type or mutated GRIN2A constructs, and performed patch-clamp recordings of transmembrane currents. The durations of channel open and closed states were significantly changed by the mutation, which could translate into disruption of excitatory responses at NMDA receptor-containing synapses.

For the third paper, the teams of Ingrid Scheffer and Heather Mefford together investigated GRIN2A mutations in 519 patients with various epileptic encephalopathies. "We set out to define the phenotypic spectrum associated with mutations in the gene," says Mefford.
Through use of exon capture and nextgeneration sequencing, the researchers identified autosomal dominant GRIN2A mutations in four patients. These individuals and their family members all had disorders that fall along the epilepsyaphasia spectrum encompassing LKS, CSWSS and autosomal dominant rolandic epilepsy-speech dyspraxia.

"Mutations in GRIN2A accounted for 9\% of the 44 patients with disorders along the epilepsy-aphasia spectrum in our cohort, and we did not find such mutations in the other 495 patients with different forms of epileptic encephalopathies," notes Mefford. Scheffer adds that "this finding is quite exciting because the epilepsy-aphasia spectrum disorders were considered likely to have an immune basis, as they improve with high-dose steroids."

Together, the findings of the three studies have important implications for diagnostic testing and our understanding of the pathogenesis of epileptic encephalopathies. Mefford, Scheffer and Szepetowski intend to search for mutations in further genes that might account for the GRIN2A-negative cases, and von Spiczak and colleagues aim to further investigate the phenotypic spectrum associated with GRIN2A mutations.

As Szepetowski notes, "GRIN2A should now be considered a crucial genetic link between different epileptic and speech disorders of the same continuum."

Katie Kingwell 